\title{
Pantelleria: isola delle donne (e della transizione ecologica?)
}

\section{Il vento ce lo disse. Donne nell'isola, di Lucia Bisi, Bolis Edizioni, Bergamo 2021}

\author{
Recensione di \\ Emilio Battisti
}

Questo è il terzo libro di Lucia Bisi che presento e nel parlarne non posso evitare di accennare anche ai due precedenti per restituire in modo più appropriato e meglio riferito i contenuti che ritengo di proporre all'attenzione.

Devo subito dire che non mi sento in grado di fare un discorso critico di carattere letterario non avendone la competenza e che cerco soprattutto di comprendere cosa questo libro può rappresentare in termini esistenziali in riferimento alla persona dell'autrice, ma anche a quella che definirei, in termini forse azzardati, un'antropologia di genere.

Per esprimere questo mio approccio faccio riferimento proprio alla dichiarazione che figura nella quarta riga della premessa al libro: «Ogni donna è protagonista di una storia e metafora di un tema generale», e mi sono interrogato su quale sia il tema generale al quale Lucia si riferisce metaforicamente, scrivendo di quelle donne.

Il primo libro che ho presentato quasi un decennio fa La Candide ovvero l'ingannevole ottimismo ${ }^{1}$, ha assunto come modello il Candide di Voltaire, e la storia si svolge a partire dagli anni '60-'70 nella Milano che cambia, così come «cambia la protagonista: maturando, ricadendo nell'ingenuità, e inseguendo illusioni che si risolvono in tragicomiche disavventure».

\footnotetext{
1 L. Bisi, La Candide. Ovvero l'ingannevole ottimismo, Canova Edizioni, Treviso 2012: https://www.arcipelagomilano.org/archives/22572.
} 
Protagonisti del suo secondo romanzo, Il giardino in salita ${ }^{2}$, sono un giardino a terrazze digradanti sulle colline di Verona e la figura del padre che quel giardino aveva creato e continua, in vecchiaia, ad accudire. Ma quel giardino è anche l'ambientazione scenica delle prime esperienze erotico sentimentali di Lucia e «delle alterne vicende con le persone che lo animano. Passato poi, alla morte del padre, nelle mani delle tre sorelle, il giardino precipita in un'insignificante freddezza che niente può consolare».

Ne La Candide, Lucia esprime l'ironica consapevolezza della casualità e causalità, strettamente intrecciate, dei fatti della vita che manifesta tutta la sua complessità nel dialettico e mai risolto rapporto tra i generi ma con piena evidenza delle figure maschili nei confronti delle quali la donna subisce una dipendenza per riscattarsi dalla quale paga un prezzo molto alto.

Nel Giardino in salita viene rivissuta la nostalgica rievocazione di un passato rappresentato dal giardino e dal padre che diventa il tramite per una rappacificazione con il genere maschile, declinata nell'affetto di parentela che sta sullo sfondo dello scenario dei gradoni di "marogne" insieme al ricordo delle prime esperienze erotiche.

Il Vento ce lo disse è la rassegnata presa d'atto delle trasformazioni che hanno spaccato in due la specie umana nei generi maschile e femminile dove il secondo sembra rappresentare l'unica opportunità per affrontare quella transizione alla quale tutti ormai alludiamo, come ultima spiaggia, per la sopravvivenza del genere umano.

È vero che quelle donne «consumano poco, non inquinano, non maltrattano il mondo, hanno vivissimo il senso del paesaggio, paesaggio che naturalmente non offendono». Ma le quindici donne nell'isola sono espressione di una condizione di rinuncia; hanno scelto l'autonomia ma sono sole, non

\footnotetext{
${ }^{2}$ L. Bisi, Il giardino in salita, Bolis Edizioni, Bergamo 2019: https://www.bolisedizioni.it lit/be_portfolio/il-giardino-in-salita.
} 
costituiscono neppure una comunità tra di loro, non hanno più rapporti con l'altro genere, sono ormai sterili e non più in grado di generare.

E le insistenti e tendenziose domande di Angelina che ricorrono nel testo, unica donna che è nata e ha sempre vissuto sull'isola e osserva il loro comportamento con il suo lungo cannocchiale, costituiscono proprio il momento della verità della loro condizione.

Di quale metafora ciascuna di loro è quindi espressione? Io credo che il messaggio forse inconsapevole che Lucia ci trasmette non è riferito a una metafora ma a un dilemma: tra un nuovo inizio del genere umano o proprio quello della sua fine.

Le donne saranno in grado di salvare il genere umano o lo accompagneranno al suo compimento? Sapranno imporre quella rivoluzione ecologica che sembra essere l'unica possibilità di salvarci oppure, assecondandone la transizione, ci indurranno a confrontarci in modo più pacato e consapevole con la prospettiva della sua fine?

Anche se il contenuto del libro si risolvesse in questa alternativa del dilemma non sarebbe affatto poco ed è quella che prediligo perché mette in conto la prospettiva della fine del genere umano così ben argomentata da Telmo Pievani nel suo libro Finitudine ${ }^{3}$.

Romanzo scientifico in cui Pievani immagina che l'incidente del 4 gennaio 1960 non sia costato la vita a Albert Camus, come effettivamente accadde, che il suo grande amico Jacques Monod vada a trovarlo in ospedale e i due dialoghino dedicando particolare attenzione al significato della fine intrinseca ad ogni realtà: dall'universo al genere umano.

Riferendomi agli altri relatori che hanno partecipato alla presentazione ${ }^{4}$, l'intervento di Gabriele Scaramuzza ha evidenziato il manifestarsi del senso di femminilità che quelle donne manifestano attraverso la cura che dedicano

\footnotetext{
3 T. Pievani, Finitudine, Raffaello Cortina Editore, Milano 2020: https://oggiscienza.it/2021 /03/13/finitudine-telmo-pievani/.

${ }^{4}$ In data 15 dicembre 2021, presso lo studio di Emilio Battisti.
} 
all'isola, il carattere fenomenologico del racconto di Lucia Bisi e ha posto all'autrice una fondamentale domanda riguardante le caratteristiche estetiche dell'isola.

Domanda alle quale ha cercato di dare risposta Costanza Rampello, figlia di Lucia, che ha rivelato come non sia tanto il vento che affascina chi approda sull'isola quanto quelle architetture costruite con blocchi di pietra lavica di carattere primigenio, roccia vulcanica prodotta da forze naturali sfuggite al controllo del genere umano.

Caratteristica che, per la conoscenza che del mediterraneo e dell'egeo, mi sembra un'esclusiva di Pantelleria, perché gli edifici delle altre isole per quanto modesti vengono abbelliti con intonachi e colori, spesso con ripetute mani di calce bianca che mitigano l'aspetto primigenio che deriva dall'esibizione dei materiali della costruzione nella loro autenticità.

Franca Pizzini ha poi fatto un resoconto, pubblicato su queste stesse pagine, molto dettagliato e utile dei contenuti del libro classificandolo come «circolare pieno di energia e di voglia di fare basate su sapienza e perseveranza». Infine si è avuta la bella e coinvolgente testimonianza di Leila Cis, ex ballerina della Scala che, dopo aver dovuto dolorosamente rinunciare alla danza, ha ripreso a ballare nei vari palcoscenici offerti dal paesaggio isolano.

Un'ultima considerazione desidero dedicare al carattere fenomenologico del racconto di Lucia Bisi che Gabriele Scaramuzza ha evidenziato e che io, privo di cultura filosofica, ho intuitivamente percepito nella "plasticità" del racconto anche perché, nel ragionarci, mi è tornata alla mente un'altra lettura per me molto interessante di Sarah Bakewell, Al caffe degli esistenzialisti. ${ }^{5}$

Quel libro mi ha fatto fare un lungo viaggio in una delle filosofie più affascinanti del XX secolo, abitata da personaggi che hanno impresso una

\footnotetext{
${ }^{5}$ S. Bakewell, Al caffè degli esistenzialisti. Libertà, Essere e Cocktail, Fazi Editore, Roma 2016: https://fazieditore.it/catalogo-libri/al-caffe-degli-esistenzialisti/.
} 
traccia indelebile nella storia del pensiero contemporaneo, da Sartre e la de Beauvoir, fino ai loro amici e fieri avversari come Camus, Heidegger, Merleau-Ponty e Lévinas e mi ha fatto capire, per l'attenzione che l'autrice dedica alle loro biografie, quanto anche le relazioni e la vita concreta di quei filosofi abbia influito sulle loro idee.

È in questi termini che ho trovato un'analogia con il libro di Lucia Bisi nel quale è chiaro ed esplicito quanto il racconto delle esperienze sue e di quelle donne sia stato in grado di dar senso ai contenuti anche filosofici che, inconsciamente, ha avuto la possibilità di esprimere.

Questo libro chiude provvisoriamente il cerchio dell'esperienza esistenziale di Lucia Bisi, questa sì, metafora del tema generale del rapporto tra i generi e della lotta delle donne per emanciparsi da svariate forme di dipendenza.

Cerchio chiuso provvisoriamente, perché sono certo che Lucia Bisi ci offrirà altri suoi contributi.

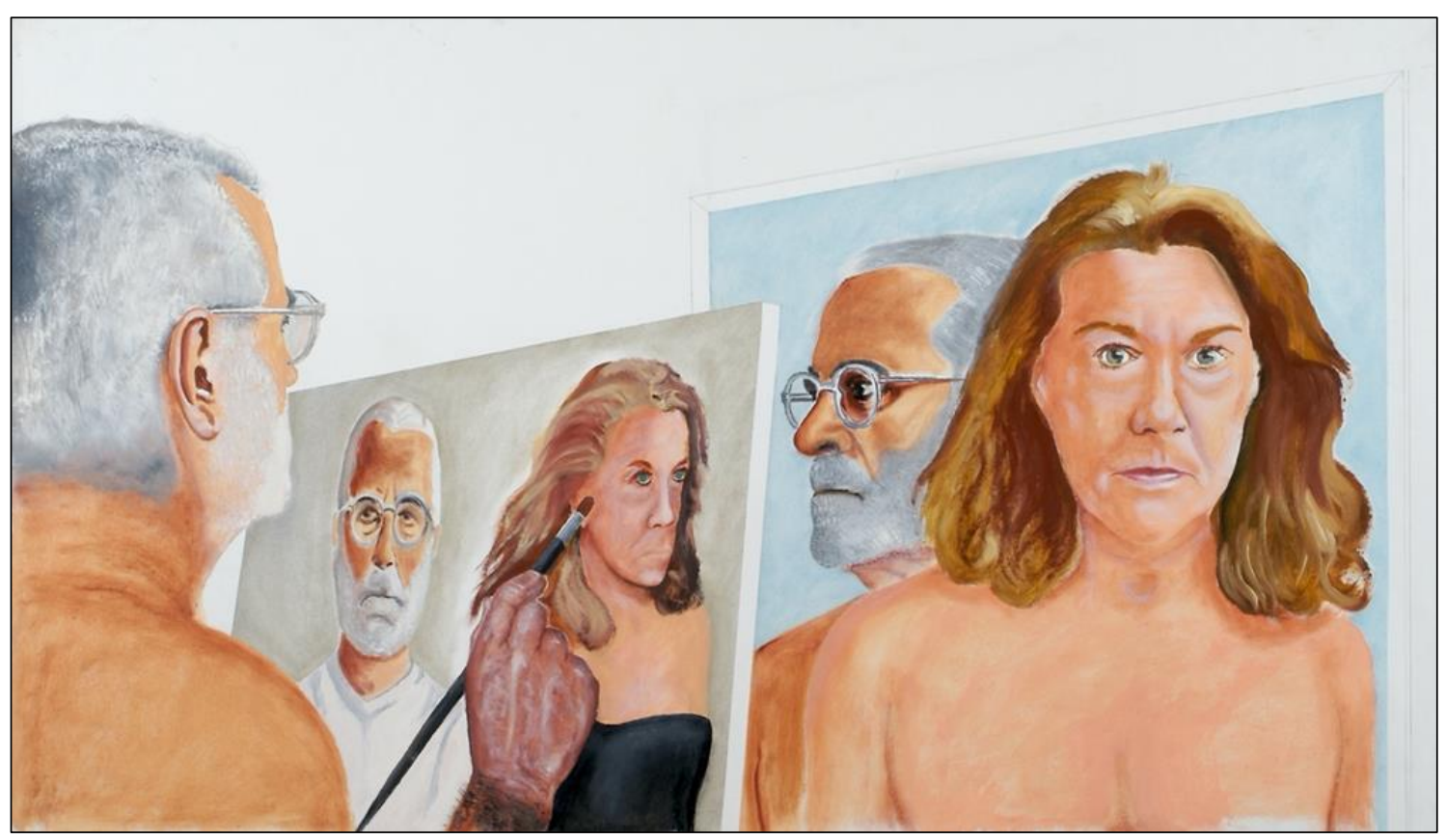

Emilio Battisti, Rispecchiamento con figura, 2006, olio su tela, 90x156. 